\title{
Powerful doping of chirality-sorted carbon nanotube films
}

\author{
Dawid Janas ${ }^{\mathrm{a}, *}$ \\ ${ }^{a}$ Department of Chemistry, Silesian University of Technology, B. Krzywoustego 4, 44-100 \\ Gliwice, Poland
}

\begin{abstract}
A fast and convenient method to create n-doped or p-doped carbon nanotube (CNT) films of improved electrical conductivity is presented herein. Free-standing thin flexible films made from unsorted CNTs and composed of predominantly $(6,5)$ or $(7,6)$ chiral angle were exposed to hydrazine $\left(\mathrm{N}_{2} \mathrm{H}_{4}\right)$ or boron trifluoride $\left(\mathrm{BF}_{3}\right)$ solutions, which decreased the sheet resistance by almost up to an order of magnitude. Analysis of the CNT films surface chemistry indicates that despite benign influence of the dopants on their composition, they strongly, but favorably affected electronic structure. Depending on the type of employed dopant, the Fermi level was strongly shifted upwards or downwards to enhance conduction of electrons or holes for employed n-doping and p-doping agents, respectively. This work provides an effective approach for the formation of CNT or graphene-based macroscopic assemblies such as films or fibers of high electrical conductivity with predetermined type of predominant charge carriers without excessive processing.
\end{abstract}

Key words: carbon nanotubes; doping; electrical conductivity

\section{Introduction}

Ever since carbon nanotubes (CNTs) and graphene have revealed their excellent electrical [13], thermal [4-6] and optical [7-9] properties, they have been the subject of intensive and extensive research all around the world. In particular, the progress in the field of portable electronics, which rapidly approaches the limits of currently used materials, has been one of

\footnotetext{
${ }^{*}$ Corresponding author. Tel/Fax: +48 32 2372958. E-mail address: dawid.janas@polsl.pl (D. Janas).
} 
the driving forces for the development of nanocarbon technology [10]. We need a next generation of conductive materials, which would be light, durable, flexible and offer much higher performance than materials used at present such as aluminium/copper (wires, interconnects) [11] or indium tin oxide (touch panels, LCDs) [12]. CNTs can already be assembled into macroscopic objects [13-16], but their performance still has to be optimized.

To improve electrical conductivity of CNTs both p-dopants (halogens [17], interhalogens [18, 19], strong acids [20]) and n-dopants (nitrogen-bearing polymers [21] or compounds [22], alkali metals [23]) can be employed. For the doping strategy to be feasible, it needs to have a strong and permanent influence on electrical conductivity of nanocarbon materials. It is especially important that the method of doping is as benign as possible and does not deteriorate the structure of individual CNTs by unnecessary functionalization.

In this paper, a convenient method is demonstrated, using which one can create n-doped or pdoped free standing CNT films. It is showed that addition of small amount of hydrazine (known for its electron-donating properties [24, 25]) or boron trifluoride can decrease the sheet resistance by up to an order of magnitude. The best results have been obtained with CNT films based on chirality sorted $(6,5)$ and $(7,6)$ material. The presented method of doping is powerful while it does not degrade the individual CNTs. To the best of my knowledge, this is the first report of using boron trifluoride as a doping agent for nanocarbon materials.

\section{Experimental}

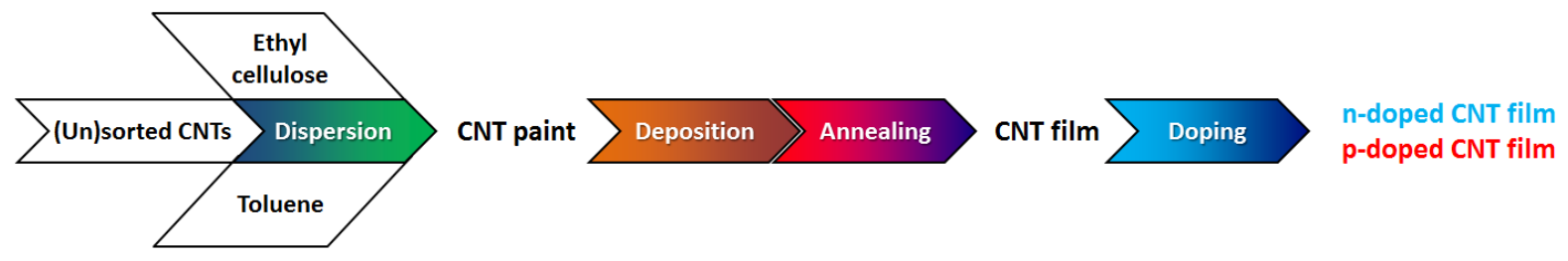

Fig. 1 Method of production of CNT films used in the study. 
The CNT films were produced by a previously reported method [26] (Fig. 1). Herein, singlewall carbon nanotubes (SWCNTs) were dispersed in toluene using ethyl cellulose. Next, CNT dispersion was deposited onto Nomex and detached from its surface upon evaporation of the solvent. Finally, binder was removed by flash annealing of the sample in air by igniting the film with a lighter for 1 second [27]. Three types of SWCNTs were used to prepare the films: unsorted Tuball ${ }^{\mathrm{TM}}$ purchased from OCSiAl and predominantly $(6,5)$ or $(7,6)$ CNTs purchased from SouthWest NanoTechnologies.

For the doping, an appropriate amount of $\mathrm{N}_{2} \mathrm{H}_{4}$ aqueous solution (50\% w/w) or $\mathrm{BH}_{3}$ methanol solution $(50 \% \mathrm{w} / \mathrm{w})$ was dripped onto the CNT films to fully soak them with the dopant. The samples were then kept in the ambient for 3 days to fully evaporate the solvent and any unabsorbed dopant.

Raman spectroscopy (inVia Raman Ramascope $\lambda=633 \mathrm{~nm}$ ) was used to probe the surface chemistry and electronic structure. 10 spectra from different positions were recorded for each sample. The given plots represent their average.

Electrical resistance measurements were carried out using a four probe method (Keithley 2000 multimeter). $10 \mu \mathrm{m} \times 30 \mathrm{~mm}$ x $30 \mathrm{~mm}$ samples were attached to custom-designed sample holders with electrical terminals made of $\mathrm{Cu}$ tape. Ag conductive paste was used to minimize the influence of contact resistance. $\mathrm{I}-\mathrm{V}$ curves were recorded up to about $100^{\circ} \mathrm{C}$ (measured by an on-line pyrometer) caused by resistive heating of the samples. A relation between specimen's temperature and sheet resistance is given to observe how permanent the doping with the increase in temperature is. 10 samples were measured for each treatment.

\section{Results}

To get an insight how dopant addition influences the electronic structure of the CNTs, Raman spectroscopy was carried out on unsorted (Fig. 2) and sorted CNT films (Fig. 3 - $(7,6)$, Fig. S1 - (6,5)). Data regarding $(6,5)$ CNT films is given in the Supplementary information 
file. Materials of very high purity in terms of $I_{D} / I_{G}$ ratios were selected to have a precise overview on the doping action. The unsorted SWCNT films had exceptionally low level of disorder $\left(\mathrm{I}_{\mathrm{D}} / \mathrm{I}_{\mathrm{G}}=0.02\right)$, which was only slightly affected by the action of $\mathrm{BF}_{3}\left(\mathrm{I}_{\mathrm{D}} / \mathrm{I}_{\mathrm{G}}=0.03\right.$, Fig. 2c) and $\mathrm{N}_{2} \mathrm{H}_{4}\left(\mathrm{I}_{\mathrm{D}} / \mathrm{I}_{\mathrm{G}}=0.03\right.$, Fig. 2e), what indicates a non-destructive character of the doping method. Most importantly, a strong charge transfer effect was observed, which gives an indirect proof that doping was successful. It was manifested by a shift of $\mathrm{G}$ or $2 \mathrm{D}$ peak position relative to the parent spectra. 


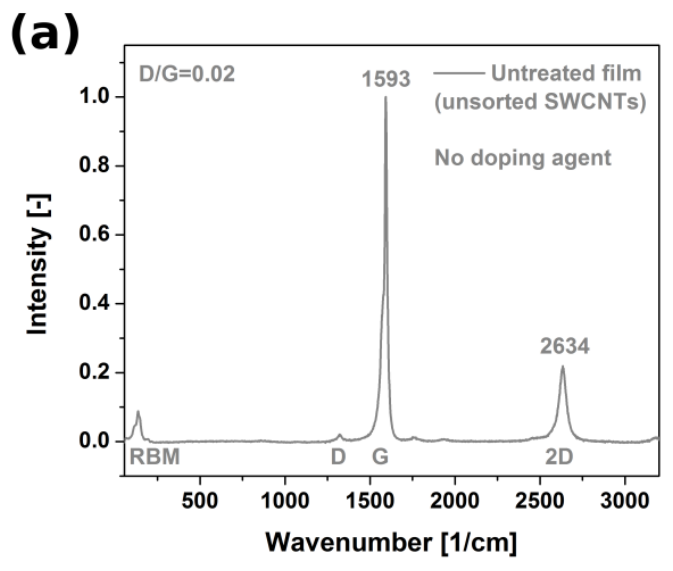

(c)

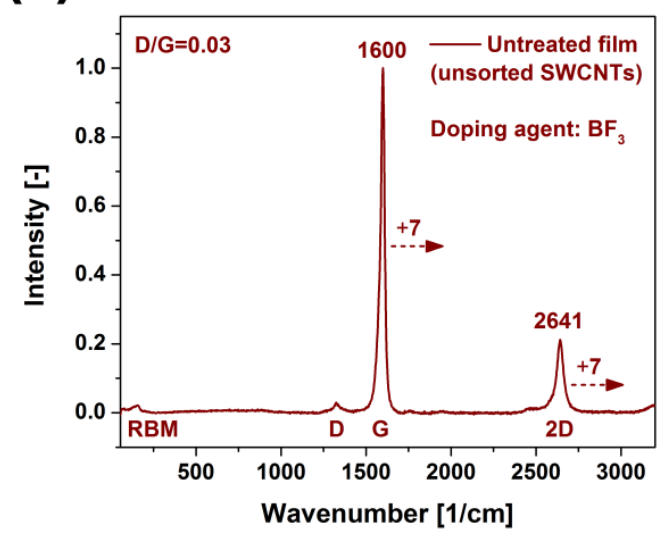

(e)

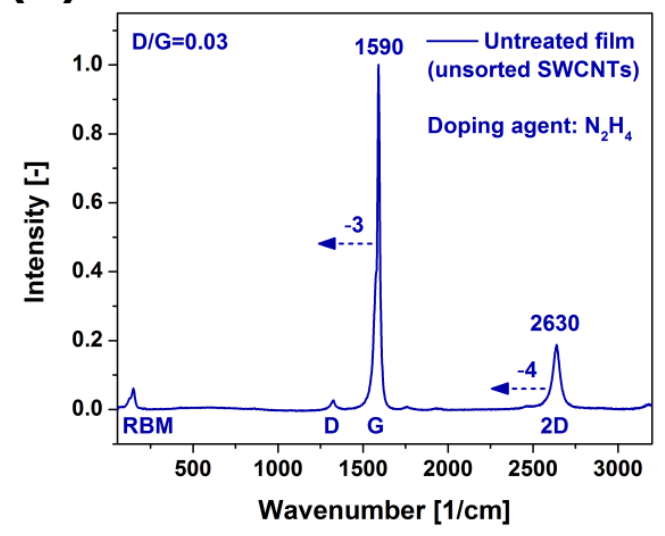

(b)

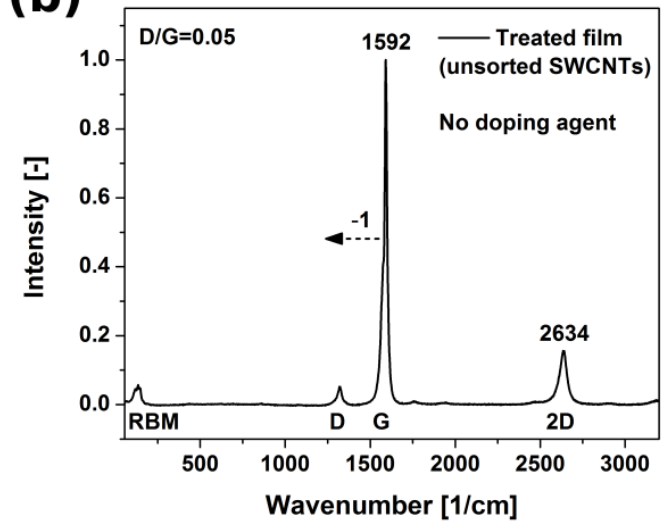

(d)

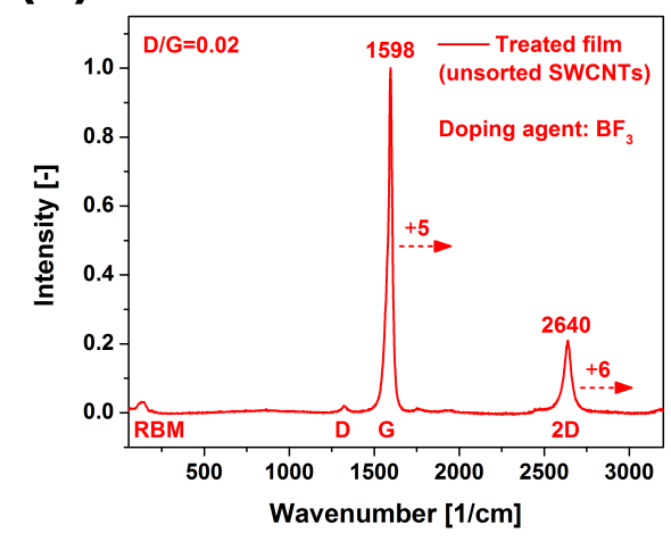

(f)

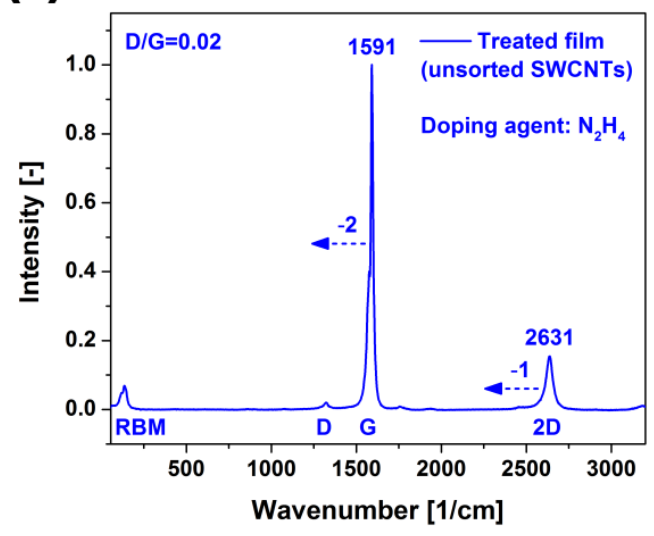

Fig. 2 Raman spectra of untreated (left column) and electrically treated to $100^{\circ} \mathrm{C}$ (right column) films made from unsorted SWCNTs. The films used were (a,b) as made, (c,d) doped with $\mathrm{BF}_{3}$ and (e,f) doped with $\mathrm{N}_{2} \mathrm{H}_{4}$.

Red-shift and blue-shifts are indicative of n-doping and p-doping, respectively $[28,29]$. In the case of unsorted SWCNT films, the peaks shifted by up to $7 \mathrm{~cm}^{-1}$ for $\mathrm{BF}_{3}$ and $4 \mathrm{~cm}^{-1}$ for $\mathrm{N}_{2} \mathrm{H}_{4}$. CNTs kept in the ambient are naturally p-doped due to the presence of oxygen [30]. As 
a consequence, the shift caused by hydrazine is smaller because it counteracts the electronwithdrawing effect of oxygen adsorbed on the surface.

Furthermore, the as-made and doped unsorted SWCNT films were heated up to $100^{\circ} \mathrm{C}$ during I-V characterization and Raman spectra were recorded again to see whether the doped material can withstand elevated temperature. It was found that electrical treatment itself causes negligible red-shift $\left(1 \mathrm{~cm}^{-1}\right.$, Fig. $\left.2 \mathrm{~b}\right)$, which slightly counteracts the action of p-doping (Fig. 2d), but reinforces n-doping (Fig. 2f). Most importantly, even after high temperature treatments the shifts remain significant, so the dopants are not desorbed under these conditions. Because unsorted material contains also metallic CNT (insensitive to doping), SWCNT films from predominantly $(6,5)$ and $(7,6)$ CNTs were manufactured and characterized (Fig. 3, Fig. S1). Materials composed of predominantly semiconducting chiralities are much more suited for this purpose.

In this case, shifts to the $\mathrm{G}$ and $2 \mathrm{D}$ peaks have also been observed and for some of the samples they were even stronger. $(7,6)$-rich SWCNT films were particularly responsive to pdoping $\mathrm{BF}_{3}$ (Fig. 3c,d). Initially, a blue-shift as large as $14 \mathrm{~cm}^{-1}$ was recorded, which decreased to $7 \mathrm{~cm}^{-1}$ after the electrical treatment, what is still a remarkable performance. $\mathrm{N}$ doping action of $\mathrm{N}_{2} \mathrm{H}_{4}$ was observed in both $(6,5)$ and $(7,6)$ SWCNT films (Fig. 3e,f and Fig. S1e,f, respectively), but again the action was not as powerful as in the case of $\mathrm{BF}_{3}$. 


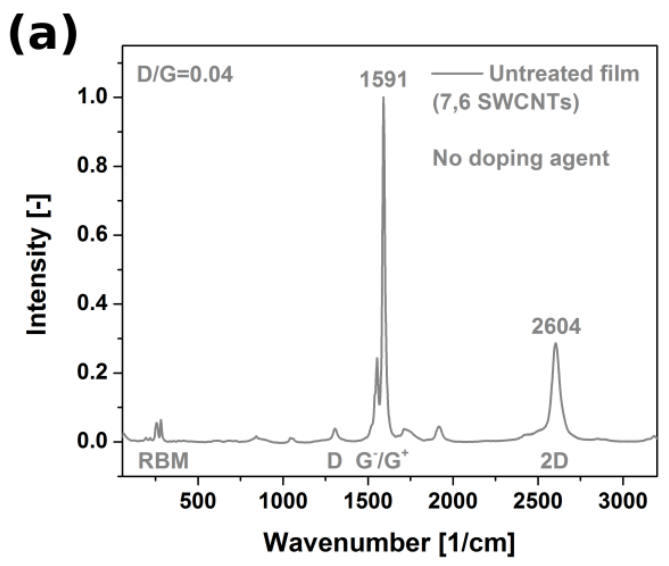

(c)

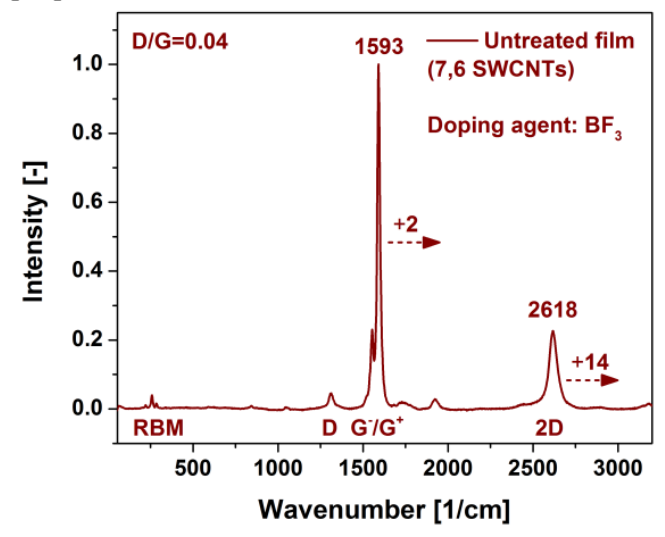

(e)

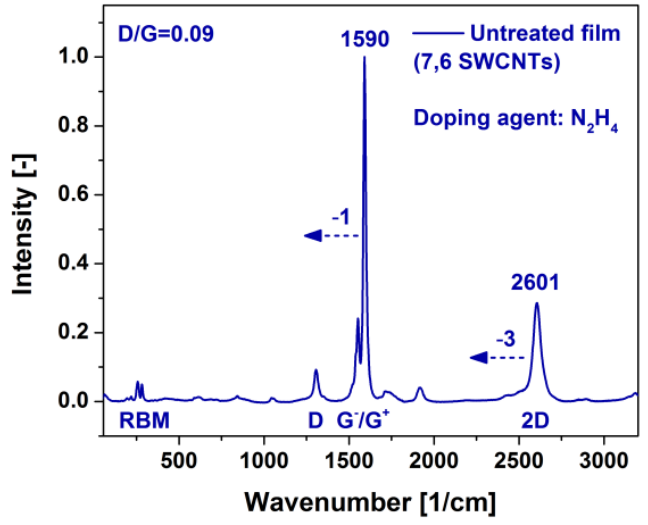

(b)

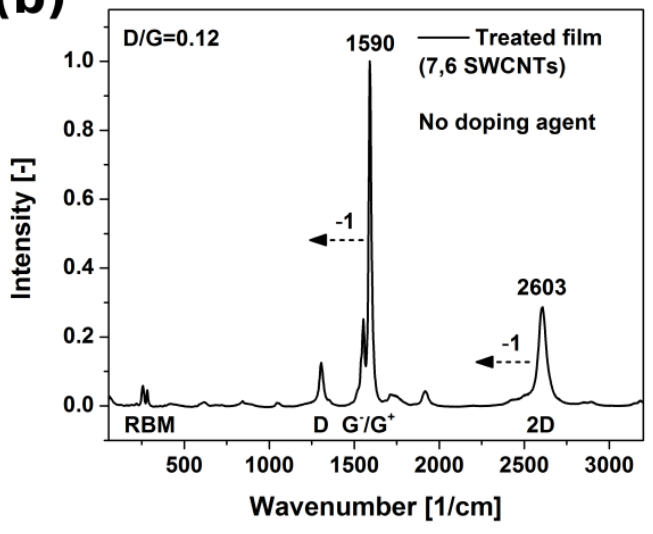

(d)

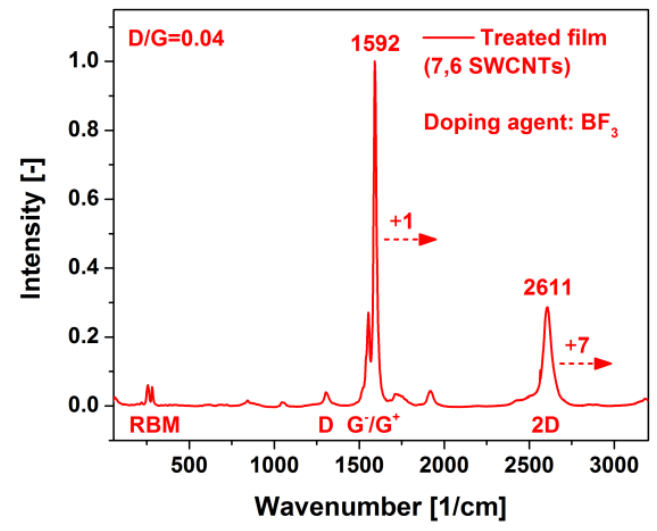

(f)

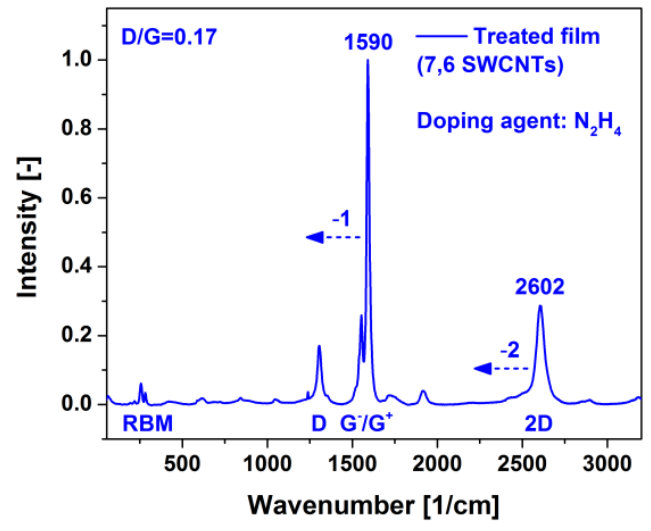

Fig. 3 Raman spectra of untreated (left column) and electrically treated to $100^{\circ} \mathrm{C}$ (right column) films made from $(7,6)$ SWCNTs. The films used were $(a, b)$ as made, $(c, d)$ doped with $\mathrm{BF}_{3}$ and (e,f) doped with $\mathrm{N}_{2} \mathrm{H}_{4}$. 
As a final step evaluation how the doping influenced electrical conductivity of the SWCNT films in terms of sheet resistance was done (Fig. $4-(7,6)$, Fig. S2 - (6,5)). Significant decrease of sheet resistance was registered, both in the case of unsorted and sorted material. Two effects are in accordance Raman spectroscopy results. Firstly, $\mathrm{BF}_{3}$ is a much stronger dopant than $\mathrm{N}_{2} \mathrm{H}_{4}$.
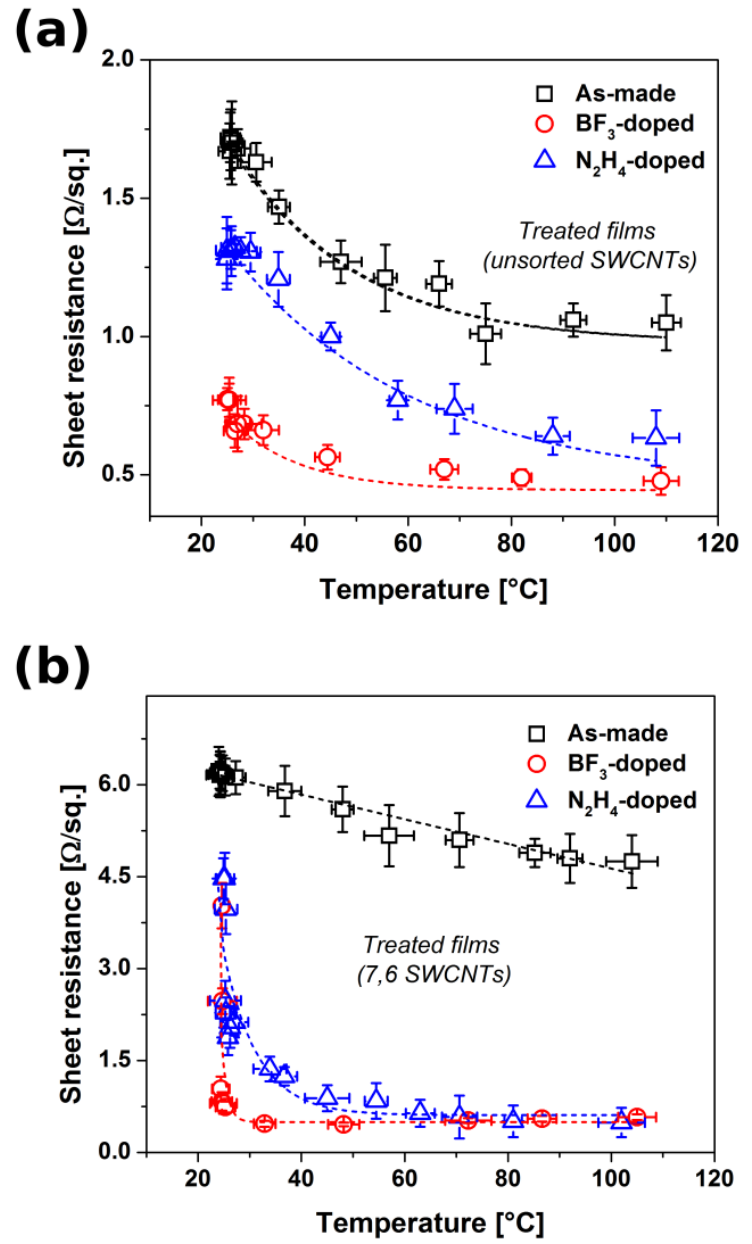

Fig. 4 Relation between temperature and sheet resistance for (a) unsorted and (b) predominantly $(7,6)$ SWCNT films. As-made as well as p/n-doped samples were characterized.

Secondly, the doping effect is more profound in the case of predominantly $(7,6)$ and $(6,5)$ SWCNT films (Fig. 4b - (7,6) and Fig. S2 - $(6,5)$ ), which are of semiconducting character 
rather than unsorted SWCNT film (Fig. 4a), which also contains metallic CNTs (as revealed by a Breit-Wigner-Fano left shoulder to the $G$ peak rather than a developed Lorentzian lineshape as in the case of $(6,5)$ and $(7,6)$ SWCNTs). Moreover, the doping was found to intensify with temperature, but in most of the cases it tended to a particular asymptotic value. The most significant decrease in sheet resistance was observed in the case of $\mathrm{BF}_{3}$ doped $(7,6)$ SWCNT film. It was lowered from 4.59 to $0.50 \Omega /$ sq (compared at about $100^{\circ} \mathrm{C}$ ), what was a reduction by almost an order of magnitude. Doping of $(6,5) \mathrm{SWCNTs}_{\text {by }} \mathrm{BF}_{3}$ resulted in similarly strong action and its sheet resistance was reduced from 3.76 to $0.47 \Omega /$ sq. In both cases the reduction was slightly smaller than in the case of $\mathrm{N}_{2} \mathrm{H}_{4}$ treatment.

\section{Conclusions}

A strategy how to prepare free-standing SWCNT films of high electrical conductivity with predetermined type of predominant charge carriers was proposed. By n-doping with $\mathrm{N}_{2} \mathrm{H}_{4}$ or p-doping with $\mathrm{BF}_{3}$ it was possible to decrease the sheet resistance of macroscopic assemblies of SWCNTs by almost an order of magnitude. What is important, such enhancement of electrical conductivity was not achieved at the expense of surface chemistry caused by unnecessary functionalization.

There is a growing need to create semiconductors with high performance for microelectronics. In particular, rapid development in the field of wearable and flexible conductors still lacks functional materials that could step in to solve the problems and circumvent the limitations of the currently used components. Nanocarbon materials such as CNTs or graphene are a viable light-weight, flexible alternative to reach the level of performance unachievable by silicon or ITO even with any further advances to their technologies. As shown by the results of this study, further progress in the preferential synthesis of particular types of CNTs coupled with appropriate methods to tune their 
electrical conductivity is a direction, which can take us closer to the application of nanocarbon devices in the real life.

\section{Acknowledgements}

D.J. thank National Science Center, Poland (under the Polonez program, grant agreement UMO-2015/19/P/ST5/03799) and the European Union's Horizon 2020 research and innovation programme (Marie Skłodowska-Curie grant agreement 665778). D.J. would also like to acknowledge Foundation for Polish Science for START scholarship ) and the Rector of the Silesian University of Technology in Gliwice for funding the research in the framework of habilitation grant (04/020/RGH17/0050). Technical assistance of Ms. Justyna Wieclawik and Ms. Marta Czernecki is also acknowledged. 


\section{References}

[1] G.J. Brady, A.J. Way, N.S. Safron, H.T. Evensen, P. Gopalan, M.S. Arnold, Quasi-ballistic carbon nanotube array transistors with current density exceeding Si and GaAs, Science Advances, 2 (2016).

[2] J.S. Friedman, A. Girdhar, R.M. Gelfand, G. Memik, H. Mohseni, A. Taflove, B.W. Wessels, J.-P. Leburton, A.V. Sahakian, Cascaded spintronic logic with low-dimensional carbon, 8 (2017) 15635.

[3] J.-H. Chen, C. Jang, S. Xiao, M. Ishigami, M.S. Fuhrer, Intrinsic and extrinsic performance limits of graphene devices on SiO2, Nat Nano, 3 (2008) 206-209.

[4] E. Pop, D. Mann, Q. Wang, K. Goodson, H. Dai, Thermal Conductance of an Individual Single-Wall Carbon Nanotube above Room Temperature, Nano Letters, 6 (2006) 96-100.

[5] A.A. Balandin, S. Ghosh, W. Bao, I. Calizo, D. Teweldebrhan, F. Miao, C.N. Lau, Superior Thermal Conductivity of Single-Layer Graphene, Nano Letters, 8 (2008) 902-907.

[6] S. Berber, Y.-K. Kwon, D. Tománek, Unusually High Thermal Conductivity of Carbon Nanotubes, Physical Review Letters, 84 (2000) 4613-4616.

[7] D. Janas, N. Czechowski, B. Krajnik, S. Mackowski, K.K. Koziol, Electroluminescence from carbon nanotube films resistively heated in air, Applied Physics Letters, 102 (2013) 181104.

[8] K.V. Sreekanth, S. Zeng, J. Shang, K.-T. Yong, T. Yu, Excitation of surface electromagnetic waves in a graphene-based Bragg grating, 2 (2012) 737.

[9] Z.-B. Liu, Y.-F. Xu, X.-Y. Zhang, X.-L. Zhang, Y.-S. Chen, J.-G. Tian, Porphyrin and Fullerene Covalently Functionalized Graphene Hybrid Materials with Large Nonlinear Optical Properties, The Journal of Physical Chemistry B, 113 (2009) 9681-9686.

[10] S. Xin, Y.-G. Guo, L.-J. Wan, Nanocarbon Networks for Advanced Rechargeable Lithium Batteries, Accounts of Chemical Research, 45 (2012) 1759-1769.

[11] P.-M. Hannula, A. Peltonen, J. Aromaa, D. Janas, M. Lundström, B.P. Wilson, K. Koziol, O. Forsén, Carbon nanotube-copper composites by electrodeposition on carbon nanotube fibers, Carbon, 107 (2016) 281-287.

[12] W.S. Koh, C.H. Gan, W.K. Phua, Y.A. Akimov, P. Bai, The Potential of Graphene as an ITO Replacement in Organic Solar Cells: An Optical Perspective, leee J Sel Top Quant, 20 (2014).

[13] F. Schütt, S. Signetti, H. Krüger, S. Röder, D. Smazna, S. Kaps, S.N. Gorb, Y.K. Mishra, N.M. Pugno, R. Adelung, Hierarchical self-entangled carbon nanotube tube networks, Nature Communications, 8 (2017) 1215.

[14] D.E. Tsentalovich, R.J. Headrick, F. Mirri, J. Hao, N. Behabtu, C.C. Young, M. Pasquali, Influence of Carbon Nanotube Characteristics on Macroscopic Fiber Properties, ACS Applied Materials \& Interfaces, 9 (2017) 36189-36198.

[15] D. Janas, S.K. Kreft, K.K.K. Koziol, Printing of highly conductive carbon nanotubes fibres from aqueous dispersion, Materials \& Design, 116 (2017) 16-20.

[16] D. Janas, K.K. Koziol, Carbon nanotube fibers and films: synthesis, applications and perspectives of the direct-spinning method, Nanoscale, 8 (2016) 19475-19490.

[17] D. Janas, S. Boncel, K.K.K. Koziol, Electrothermal halogenation of carbon nanotube films, Carbon, 73 (2014) 259-266.

[18] D. Janas, A.P. Herman, S. Boncel, K.K.K. Koziol, lodine monochloride as a powerful enhancer of electrical conductivity of carbon nanotube wires, Carbon, 73 (2014) 225-233.

[19] D. Janas, K.Z. Milowska, P.D. Bristowe, K.K.K. Koziol, Improving the electrical properties of carbon nanotubes with interhalogen compounds, Nanoscale, 9 (2017) 3212-3221.

[20] D. Janas, A.C. Vilatela, K.K.K. Koziol, Performance of carbon nanotube wires in extreme conditions, Carbon, 62 (2013) 438-446.

[21] M. Shim, A. Javey, N.W. Shi Kam, H. Dai, Polymer Functionalization for Air-Stable n-Type Carbon Nanotube Field-Effect Transistors, Journal of the American Chemical Society, 123 (2001) 1151211513. 
[22] J.-L. Xu, R.-X. Dai, Y. Xin, Y.-L. Sun, X. Li, Y.-X. Yu, L. Xiang, D. Xie, S.-D. Wang, T.-L. Ren, Efficient and Reversible Electron Doping of Semiconductor-Enriched Single-Walled Carbon Nanotubes by Using Decamethylcobaltocene, Scientific Reports, 7 (2017) 6751.

[23] R.S. Lee, H.J. Kim, J.E. Fischer, A. Thess, R.E. Smalley, Conductivity enhancement in single-walled carbon nanotube bundles doped with $\mathrm{K}$ and $\mathrm{Br}$, Nature, 388 (1997) 255-257.

[24] P.-C. Wang, Y.-C. Liao, Y.-L. Lai, Y.-C. Lin, C.-Y. Su, C.-H. Tsai, Y.-J. Hsu, Conversion of pristine and $\mathrm{p}$-doped sulfuric-acid-treated single-walled carbon nanotubes to $\mathrm{n}$-type materials by a facile hydrazine vapor exposure process, Materials Chemistry and Physics, 134 (2012) 325-332.

[25] D. Ruixuan, X. Dan, X. Jianlong, S. Yilin, S. MengXing, Z. Cheng, L. Xian, Adjustable hydrazine modulation of single-wall carbon nanotube network field effect transistors from $p$-type to $n$-type, Nanotechnology, 27 (2016) 445203.

[26] D. Janas, M. Rdest, K. Koziol, Free-standing films from chirality-controlled carbon nanotubes, Materials \& Design, (in press) (2017).

[27] D. Janas, G. Stando, Unexpectedly strong hydrophilic character of free-standing thin films from carbon nanotubes, Scientific Reports, 7 (2017) 12274.

[28] D. Janas, S. Boncel, A.A. Marek, K.K. Koziol, A facile method to tune electronic properties of carbon nanotube films, Materials Letters, 106 (2013) 137-140.

[29] W.S. Jang, S.S. Chae, S.J. Lee, K.M. Song, H.K. Baik, Improved electrical conductivity of a noncovalently dispersed graphene-carbon nanotube film by chemical p-type doping, Carbon, 50 (2012) 943-951.

[30] A. Lekawa-Raus, L. Kurzepa, G. Kozlowski, S.C. Hopkins, M. Wozniak, D. Lukawski, B.A. Glowacki, K.K. Koziol, Influence of atmospheric water vapour on electrical performance of carbon nanotube fibres, Carbon, 87 (2015) 18-28. 